\title{
Partial response to sorafenib treatment associated with transient grade 3 thrombocytopenia in a patient with locally advanced thyroid cancer
}

Fabián Pitoia', Erika Abelleira', Fernando Jerkovich', Carolina Urciuoli', Graciela Cross' ${ }^{1}$

\begin{abstract}
SUMMARY
Advanced radioactive refractory and progressive or symptomatic differentiated thyroid carcinoma (DTC) is a rare condition. Sorafenib was recently approved for the treatment of these patients. We present the case of a 67 year old woman diagnosed with DTC who underwent a total thyroidectomy with central, lateral-compartment neck dissection and shaving of the trachea and esophagus due to tumor infiltration. A local recurrence was detected 14 months later requiring, additionally, two tracheal rings resection. The patient received a cumulative ${ }^{131} \mathrm{I}$ dose of $650 \mathrm{mCi}$ and developed dysphagia and dyspnea 63 months after initial surgery. A ${ }^{18} \mathrm{FGD}-\mathrm{PET} / \mathrm{CT}$ showed progression of the local mass associated to hypermetabolic pulmonary nodules. Sorafenib $800 \mathrm{mg} /$ day was then prescribed. A dose reduction to $400 \mathrm{mg} /$ day was necessary due to grade 3 thrombocytopenia that appeared four months after drug prescription. Platelet count went to normal after this dose reduction. Five months after initiation of sorafenib, a partial response of the local mass with significant intra-tumoral necrosis was observed. We conclude that sorafenib is a valid option for locally advanced DTC and that the platelet count should be evaluated regularly because it seems that thrombocytopenia might be more frequently observed in DTC than in other types of tumors. Arch Endocrinol Metab. 2015;59(4):347-50
\end{abstract}

${ }^{1}$ División de Endocrinología, Hospital de Clínicas, Universidad de Buenos Aires, Buenos Aires, Argentina

Correspondence to: Fabián Pitoia División Endocrinología, Hospital de Clínicas,

Universidad de Buenos Aires Córdoba, 2351, $5^{\circ}$ piso 1001 - Buenos Aires, Argentina fpitoia@intramed.net

Received on Mar/17/2015 Accepted on Apr/22/2015 DOI: 10.1590/2359-3997000000059

\section{Keywords}

Thyroid cancer; sorafenib; thrombocytopenia

\section{INTRODUCTION}

$\mathrm{T}$ hyroid carcinoma is a rare disease. It accounts for approximately $1 \%$ of all new malignant disease, including nearly $0.5 \%$ of cancers in men and $1.5 \%$ of cancers in women (1). Differentiated thyroid carcinoma is usually treated with total thyroidectomy and postoperative levothyroxine for thyroid stimulating hormone suppression $(2,3)$. Depending mainly on the risk of recurrence, ablation of the thyroid remnant with radioactive iodine 131 (RAI) is added to the standard treatment $(2,3)$.

This strategy, together with additional RAI doses or external beam radiotherapy in isolated cases, is associated with an overall survival rate of approximately $90 \%$ at 20 years (4). The prognosis remains relatively favorable even among patients who experience recurrent disease (5). Treatment of distant metastases includes RAI that can be administered several times. However, once DTC is no longer amenable to RAI therapy or surgery, expected survival declines rapidly (2-4), and death from thyroid carcinoma within 3 years under these circumstances is common (6-8). RAI refractory DTC is an advanced cancer that occurs in patients who have at least one tumor focus without radioactive iodine uptake, or by progression of the disease within one year after RAI treatment, and/or in patients with persistent disease after administration of a cumulative radioactivity of 22 $\mathrm{GBq}(600 \mathrm{mCi})(9)$. RAI-refractory DTC is rare with an estimated incidence of 4 cases per million population per year (4). A number of systemic therapies have been evaluated in Phase II or III trials and some of these systemic therapies are recommended for patients with RAI-refractory DTC in treatment guidelines $(2,3)$.

Sorafenib is a member of the tyrosine kinase inhibitors. In vitro, it has shown to inhibit multiple kinases thought to be involved in both cell proliferation and angiogenesis. Sorafenib was recently approved in US, Europe and some other countries for the treatment of RAIrefractory and progressive advanced thyroid cancer after 
the presentation of the DECISION trial which showed that progression-free survival was significantly improved with sorafenib $(\mathrm{n}=207)$ compared with placebo $(\mathrm{n}=$ 210) (HR 0.587; 95\% CI 0.454-0.758; p < 0.0001; median 10.8 versus 5.8 months, respectively) (10).

The aim of this publication is to show the case of one patient with advanced, progressive and symptomatic RAI-refractory thyroid cancer who received sorafenib treatment and showed a partial response during the first 5 months of treatment and presented with an atypical adverse event (thrombocytopenia) which was solved with dose reduction of the drug. Written consent was obtained from the patient and our ethical committee.

\section{CASE REPORT}

A 67 year-old woman was diagnosed with papillary thyroid carcinoma after a fine needle aspiration performed in October 2007. A left lobectomy was first indicated due to tumor fixation to the surrounding structures. One year after the diagnosis, she was referred to our University Hospital. A completion thyroidectomy with central and lateral-compartment neck dissection (levels II, III, IV and V) and shaving of the trachea and esophagus for superficial tumor invasion were carried out. The pathological examination revealed a papillary thyroid carcinoma classic variant measuring $8 \mathrm{~cm}$ in bigger diameter with extra-thyroidal extension. No evidence of lymph node metastasis in 27 resected lymph nodes were observed. A local recurrence was detected 14 months later. Resection of persistent central compartment lymph nodes ( 2 of 6 with metastasis and extra-nodal extension) and two tracheal rings was carried out at that moment. The patient received a cumulative activity of $650 \mathrm{mCi}^{131} \mathrm{I}$ in a period of 21 months. During the 75 months of follow-up, the serum thyroglobulin was undetectable due to the presence of antithyroglobulin antibodies. Only a thyroid bed uptake was observed after performing the post-dose whole body scans. Forty-three months after initial surgery, a ${ }^{18}$ Fluorodeoxyglucose Positron Emission Tomography $\left({ }^{18} \mathrm{FDG} \mathrm{PET} / \mathrm{CT}\right)$ was indicated showing a local hypermetabolic nodule of $1.7 \mathrm{~cm}$ in bigger diameter with a maximum Standardized Uptake Value (SUV max) of 4.5. A compression and deviation of the trachea and esophagus and bilateral hypermetabolic pulmonary nodules (SUV max 2) were also observed (Figure 1). The patient developed dysphagia and dyspnea 20 months later. A new ${ }^{18}$ FDG PET/CT revealed progression in the local mass size and increase in FDG uptake $(5.8 \mathrm{~cm}$ and SUV max 6.9) and in the $1.4 \mathrm{~cm}$ hypermetabolic pulmonary nodule in the upper right lobe (SUV $\max 8.3$ ) (Figure 2). Due to radioiodine-refractory, progressive and symptomatic thyroid cancer, Sorafenib $800 \mathrm{mg} /$ day was prescribed. Due to the fact that the patient could not swallow the pills, we recommended that they were diluted in $60 \mathrm{ml}$ with an additional $180 \mathrm{~mL}$ of water swallowed as a rinse of the dosing vessel as previously suggested (11).

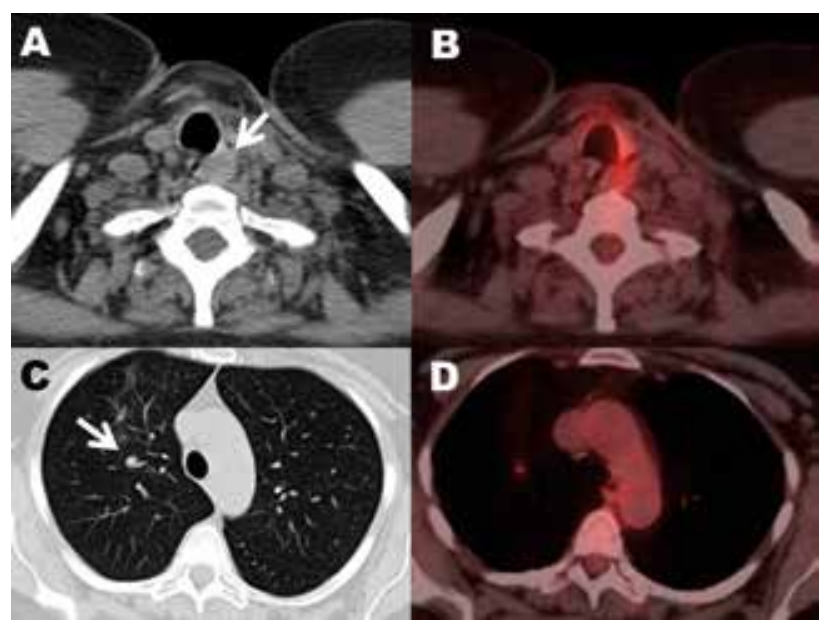

Figure 1. Images performed forty-three months after initial surgery. (A) Computed tomography (CT) scan, axial view, showing a nodule of $1.7 \mathrm{~cm}$ in bigger diameter compressing and deviating the trachea (arrow). (B) The ${ }^{18}$ Fluorodeoxyglucose Positron Emission Tomography $\left({ }^{18} \mathrm{FDG}\right.$ PET/CT) of the same image reveals a maximum Standardized Uptake Value (SUV max) of 4.5. (C) CT scan and (D) ${ }^{18}$ FDG PET/CT, axial view, detecting a pulmonary nodule less than $1 \mathrm{~cm}$ in the upper right lobe (arrow) with an SUV max of 2. $254 \times 190 \mathrm{~mm}(96 \times 96 \mathrm{DPI})$.

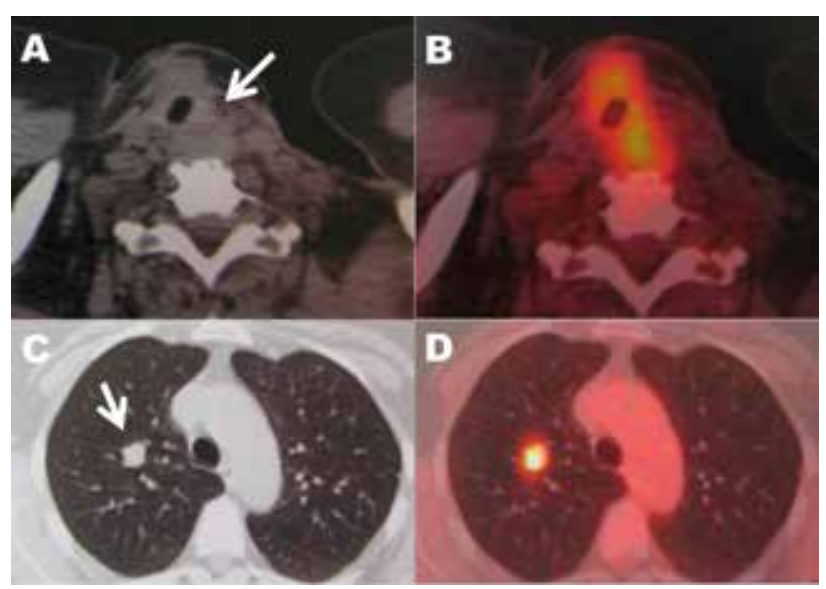

Figure 2. Images performed sixty-six months after initial surgery. (A) CT scan and (B) ${ }^{18}$ FDG PET/CT, axial view, showing a neck local mass of 5.8 $\mathrm{cm}$ in size (arrow) with an SUV max of 6.9. (C) CT scan and (D) ${ }^{18} \mathrm{FDG} \mathrm{PET/}$ CT revealing a hypermetabolic pulmonary nodule (arrow) of $1.4 \mathrm{~cm}$ size in the upper right lobe (SUV max 8.3). $238 \times 167 \mathrm{~mm}$ (96 x 96 DPI). 
After one month of treatment, the following adverse effects were observed: diarrhea, stomatitis, alopecia, hypertension, loss of weight (all of them grade 1 ) and grade 2 hand-foot skin reaction. Sorafenib withdrawal was necessary due to grade 3 thrombocytopenia $\left(25,000 \times 10^{3} / \mathrm{mm}^{3}\right)$ which appeared four months after treatment initiation. The patient presented subconjunctival hemorrhage with spontaneous resolution. No other bleeding manifestations were detected. Two weeks after sorafenib withdrawal, the platelet count was again in the normal range. After two months of resuming sorafenib $400 \mathrm{mg} /$ day treatment, no evidence of thrombocytopenia was observed. No other bone marrow cell linages reductions were observed (Table 1).

Five months after the initiation of sorafenib therapy, a CT scan demonstrated a reduction from $5.8 \mathrm{~cm}$ to $3.8 \mathrm{~cm}$ in the local mass with significant intra-tumoral necrosiss, and a decrease from $1.4 \mathrm{~cm}$ to $1.2 \mathrm{~cm}$ in the lung metastasis (Figure 3). This represents a $30.6 \%$ decrease in the

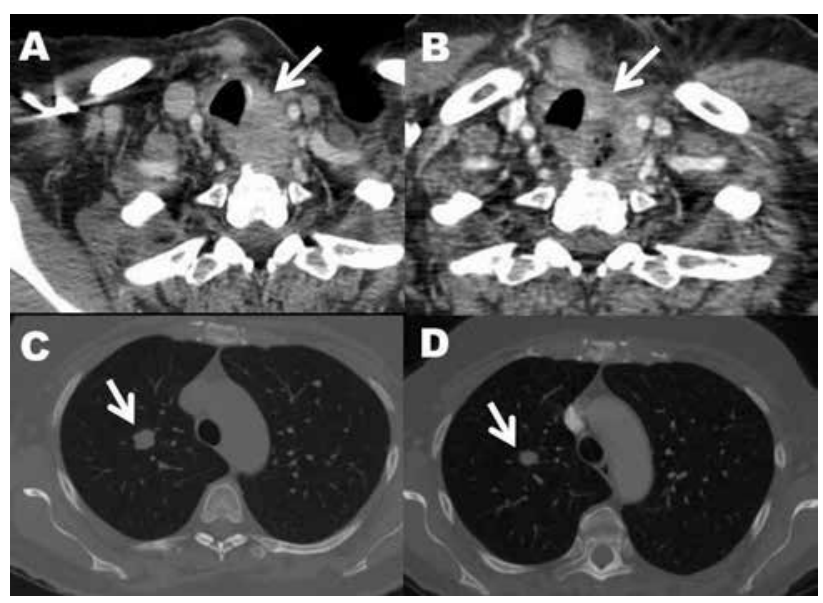

Figure 3. Comparison between CT, axial view, prior to treatment ( $\mathbf{A}$ and $\mathbf{B}$ ) and 5 months after sorafenib initiation (C and $\mathbf{D})$. Imaging (A) shows a local mass of $5.8 \mathrm{~cm}$ in maximum diameter (arrow) and (C) reveals a pulmonary nodule of $1.4 \mathrm{~cm}$ size in the upper right lobe (arrow). Imaging (B) demonstrates a reduction in the local mass to $5.0 \mathrm{~cm}$ in maximum diameter with significant intra-tumoral necrosis of $1.2 \mathrm{~cm}$ in size $(3.8 \mathrm{~cm}$ in longest diameter of the mass excluding the area of necrosis) and (D) shows a reduction in the pulmonary nodule to $1.2 \mathrm{~cm}$ in size. $254 \times 182$ $\mathrm{mm}(96 \times 96 \mathrm{DPI})$. sum of the diameters of the target lesions, which means a partial response to treatment according to mRECIST criteria. The obstructive symptoms completely resolved.

\section{DISCUSSION}

Sorafenib is a multiple tyrosine kinase inhibitor (TKI), targeting CRAF, BRAF, VEGF receptor (VEGFR)-1, $-2,-3$, PDGF receptor (PDGFR)- $\beta$, RET, $c$-kit and Flt-3 (10). As a multifunctional inhibitor, sorafenib inhibits tumor growth, progression, metastasis and angiogenesis, as well as downregulating mechanisms that protect tumors from apoptosis.

In approximately $2 / 3$ of the patients from the DECISION trial (in half of patients in an off-label published study of one of the authors; PF) (12), patients required dose reductions due to adverse events (AEs) that were mostly grade 1 or 2 . The most common AEs have been hand-foot skin reactions and other skin toxicities, fatigue, weight loss, and diarrhea, as it was observed in this patient. However, thrombocitopenia is a rare $\mathrm{AE}$ presented in patients being treated with sorafenib.

In hepatocellular carcinoma (SHARP study), the frequency of grade 3-4 thrombocitopenia was only $4 \%$ for sorafenib vs. $1 \%$ for the placebo group (13). On the other side, in renal cell carcinoma (TARGET Study), this AE occurred in only $1 \%$ of the patients treated with sorafenib vs. $0 \%$ for the placebo group (14).

In the DECISION trial the frequency of thrombocitopenia was $18.4 \%$ for the Sorafenib arm vs. $9.6 \%$ in the placebo group. In none of the patients who presented this $\mathrm{AE}$ it was grade 3 (between 50,000 and 25,001 x $\left.10^{3} / \mathrm{mm}^{3}\right)$ or grade $4\left(<25,000 \times 10^{3} / \mathrm{mm}^{3}\right)$. Thrombocytopenia was not an $\mathrm{AE}$ which led to a dose decreasing or dose interruption in any patient of the DECISION trial (10). Nevertheless, in this patient, it seems that this $\mathrm{AE}$ was dose dependent because the patient resumed her platelet count to normal after dose reduction to $400 \mathrm{mg} /$ day.

Table 1. Platelet count, white blood cell (WBC) count and hemoglobin values ( $\mathrm{Hb})$ during sorafenib therapy

\begin{tabular}{|c|c|c|c|c|c|c|c|c|}
\hline & Baseline & $1 \mathrm{mo}$ & $4 \mathrm{mo}$ & $\begin{array}{c}4 \mathrm{mo} \\
+1 \mathrm{wk} \\
\end{array}$ & $5 \mathrm{mo}$ & $\begin{array}{c}5 \mathrm{mo} \\
+2 \mathrm{wk} \\
\end{array}$ & $\begin{array}{r}5 \mathrm{mo} \\
+3 \mathrm{wk} \\
\end{array}$ & $6 \mathrm{mo}$ \\
\hline Platelets $\left(\times 10^{3} / \mathrm{mm}^{3}\right)$ & 158 & 107 & $25^{*}$ & $173^{\star \star}$ & 242 & 151 & 110 & 130 \\
\hline WBC $\left(x 10^{3} / \mathrm{mm}^{3}\right)$ & 4.5 & 4.3 & 3.7 & 5.4 & 4.4 & 4.9 & 4.3 & 6.0 \\
\hline $\mathrm{Hb}(\mathrm{g} / \mathrm{dL})$ & 14.0 & 14.3 & 13.2 & 11.5 & 10.8 & 13 & 13.1 & 13.7 \\
\hline
\end{tabular}

Mo.: month/s; Wk.: week/s; ${ }^{*}$ Sorafenib withdrawal; ** Resumption of sorafenib treatment with $400 \mathrm{mg} /$ day (half of the initial dose); normal values (NV) for platelets: 150 to $350 \times 10^{3} / \mathrm{mm}^{3}$; NV for WBC: 3.7 to 10.1 ; NV for $\mathrm{Hb} 12.0$ to 16.0 . 
We think that the higher prevalence of thrombocytopenia in patients with advanced thyroid cancer probably could be related to the cumulative damage of the bone marrow that is usually observed in these patients who generally have a cumulative activity of radioiodine higher than $600 \mathrm{mCi}$, as it occurred in our patient. Padovani and cols. (15) observed a small but statistically significant decrease in hemoglobin values and platelet count one year after the last RAI treatment in patients with metastatic DTC who had received at least 250 $\mathrm{mCi}^{131}$ I during their follow-up. On the other side, an increased risk of leukaemias was found with increasing cumulative activity of ${ }^{131} \mathrm{I}$ administered (16). However, more studies are needed to assess the clinical implications of the RAI cumulative activities effects on bone marrow.

We conclude that this patient with locally advanced thyroid cancer had a relative fast response after sorafenib treatment and that it should be considered as a valid option for patients with progressive and/or symptomatic local disease which is not amenable for other treatments. The platelet count should be evaluated regularly after sorafenib prescription because it seems that thrombocytopenia might be more frequently observed in DTC than in other types of tumors.

Disclosure: Fabián Pitoia is a consultant and speaker bureau for Bayer. The remaining authors have nothing to declare.

\section{REFERENCES}

1. Sherman SI. Thyroid carcinoma. Lancet. 2003;361(9356):501-11.

2. American Thyroid Association (ATA) Guidelines Taskforce on Thyroid Nodules and Differentiated Thyroid Cancer; Cooper DS, Doherty GM, Haugen BR, Kloos RT, Lee SL, Mandel SJ, et al. Revised American Thyroid Association management guidelines for patients with thyroid nodules and differentiated thyroid cancer. Thyroid. 2009;19(11):1167-214.

3. Pitoia F, Califano I, Vázquez A, Faure E, Gauna A, Orlandi A, et al. Inter Society Consensus for the Management of Patients with Differentiated Thyroid Cancer. Rev Arg Endocrinol Metab. 2014;51:85-118.

4. Brenner $\mathrm{H}$. Long-term survival rates of cancer patients achieved by the end of the 20th century: a period analysis. Lancet. 2002;360:1131-5.

5. Mazzaferri EL, Jhiang SM. Long-term impact of initial surgical and medical therapy on papillary and follicular thyroid cancer. Am J Med. 1994;97(5):418-28.

6. Durante C, Haddy N, Baudin E, Leboulleux S, Hartl D, Travagli $\mathrm{JP}$, et al. Long-term outcome of 444 patients with distant metastases from papillary and follicular thyroid carcinoma: benefits and limits of radioiodine therapy. J Clin Endocrinol Metab. 2006;91(8):2892-9.

7. Ruegemer JJ, Hay ID, Bergstralh EJ, Ryan JJ, Offord KP, Gorman CA. Distant metastases in differentiated thyroid carcinoma: a multivariate analysis of prognostic variables. J Clin Endocrinol Metab. 1988;67(3):501-8.

8. Shoup M, Stojadinovic A, Nissan A, Ghossein RA, Freedman S, Brennan MF, et al. Prognostic indicators of outcomes in patients with distant metastases from differentiated thyroid carcinoma. J Am Coll Surg. 2003;197(2):191-7.

9. Schlumberger M, Brose M, Elisei R, Leboulleux S, Luster M, Pitoia $F$, et al. Definition and management of radioactive iodine-refractory differentiated thyroid cancer. Lancet Diabetes Endocrinol. 2014;2(5):356-8.

10. Brose MS, Nutting CM, Jarzab B, Elisei R, Siena S, Bastholt L, et al.; DECISION investigators. Sorafenib in radioactive iodinerefractory, locally advanced or metastatic differentiated thyroid cancer: a randomised, double-blind, phase 3 trial. Lancet. 2014;384(9940):319-28.

11. Lettieri JT, Dubowy R, Xia C, Rotolo C, Zinny MA. Bioavailability of sorafenib tablets administered as a liquid suspension. $J$ Clin Oncol. 2009. ASCO Annual Meeting Proceedings; Abstract: e14549.

12. Pitoia F. Response to sorafenib treatment in advanced metastatic thyroid cancer. Arq Bras Endocrinol Metabol. 2014;58(1):37-41.

13. Llovet JM, Ricci S, Mazzaferro V, Hilgard P, Gane E, Blanc JF, et al.; SHARP Investigators Study Group. Sorafenib in advanced hepatocellular carcinoma. N Engl J Med. 2008;359:378-90.

14. Escudier B, Eisen T, Stadler WM, Szczylik C, Oudard S, Siebels $M$, et al.; TARGET Study Group. Sorafenib in advanced clear-cell renal-cell carcinoma. N Engl J Med. 2007;356(2):125-34.

15. Padovani RP, Tuttle RM, Grewal R, Larson SM, Boucai L. Complete blood counts are frequently abnormal 1 year after dosimetryguided radioactive iodine therapy for metastatic thyroid cancer. Endocr Pract. 2014;20(3):213-20.

16. Rubino C, de Vathaire F, Dottorini ME, Hall P, Schvartz C, Couette $\mathrm{JE}$, et al. Second primary malignancies in thyroid cancer patients. Br J Cancer. 2003;89(9):1638-44. 